\title{
Energy-Delay Optimization in an Asynchronous Sensor Network with Multiple Gateways
}

\author{
Reuven Cohen and Boris Kapchits \\ Department of Computer Science \\ Technion \\ Haifa Israel
}

\begin{abstract}
This paper studies the problem of energy efficient routing in a sensor network with multiple gateways. Due to the complexity of this problem, we divide it into two subproblems: the problem of constructing efficient routing trees and the problem of wake-up frequency assignment in a network with multiple routing trees. For the first problem we present an optimal algorithm and an approximation algorithm that achieves very close performance but can be more easily implemented. We prove that the second problem is NP-hard and propose a polynomial time approximation algorithm.
\end{abstract}

\section{INTRODUCTION}

We consider a mesh sensor network, with hundreds of simple sensors that are densely deployed at some inspected site and report to a centralized gateway. Since most of the sensors do not have direct wireless communication with the gateway, they route the packets through other sensors. There are many approaches for routing in sensor networks. In this work we assume that routing paths towards the gateways are constructed by the nodes in advance.

To minimize idle listening, which is the main source for energy waste, the sensors turn their communication hardware on and off. In order for two neighboring sensors to communicate, both must be active at the same time. There are two main approaches to scheduling simultaneous wake-up of neighboring sensors: global or local synchronization [1]-[4]. Since global synchronization is inefficient and very difficult to achieve in big networks [2]-[4], we consider an asynchronous network where sensor wake-ups are only synchronized locally. That is, each node selects its wake-up schedule and informs neighboring nodes about its selection. A node that needs to send a packet through a neighbor must wake up and transmit it during the neighbor's duty cycle. This communication model imposes a clear tradeoff between the delay encountered by a packet routed along the sensor network and the time during which the sensors along the route are in active mode. This tradeoff was studied in several works [5]-[7] .

In [7], the tradeoff between packet latency and energy expenditure in asynchronous sensor networks is studied. It is assumed that a single route is used from each sensor to the gateway. This implies that the collection of routes from the nodes to the gateway defines a directed tree rooted at the network's gateway. This is a conventional approach in many "simple" data networks, where selecting different routes for different flows between two nodes is too complicated. Since no assumption is made regarding how routes are selected, the schemes proposed in [8], [9], as well as in many other papers, are all applicable. Following this assumption, each sensor node has a designated neighbor (parent) to which it forwards packets destined for the gateway. These packets originate at the node itself or at some other node.

Energy expenditure is commonly assumed to be governed not by the amount of transmitted data but by the time the sensors spend in active mode [7], [10], [11]. This is true, for example, in alarm sensor networks, where the traffic is very low while the latency requirements are high. Thus, there is a clear tradeoff between energy expenditure and the end-to-end delay. It is shown in [7] that the energy expenditure and the end-to-end delay are both governed by the duty cycle of the nodes, which is defined by their wake-up frequency. Hence, the problem of energy-latency tradeoff is solved in [7] by finding an optimal assignment of wake-up frequency to nodes. This assignment guarantees an upper bound on the maximum endto-end delay, while minimizing the total expended energy and bounding the energy expended by every node. Throughout the paper this assignment is denoted as the $\tau / D$-optimal wake-up frequency assignment, where $\tau$ is the bound on the energy expended by the nodes and $D$ is the bound on the end-to-end delay. This energy expenditure model is explained in greater detail in Section III.

The contribution of this paper is two-fold. First, instead of solving the sensor wake-up scheduling problem for a single routing tree rooted at a single gateway [7], we solve it for multiple trees rooted at one or more gateways. The benefits of using multiple trees are clear: (a) faster recovery from gateway failures and (b) load sharing [12], [13] . We present both optimal and approximation algorithms for the considered problem. The optimal algorithm cannot be easily implemented in a distributed sensor networks, and it is mainly used as a benchmark. While the approximation algorithm does not find the optimal solution, it is both efficient and simple for distributed implementation. Such an implementation requires a low message complexity of $O\left(n|V|^{2}\right)$, where $n$ is the number of spanning trees and $|V|$ is the number of nodes. The second contribution of the paper is addressing the tree building problem from the perspective of the optimal wake-up frequency assignment. In other words, the algorithm for building the tree takes into account the optimization criterion imposed by the optimal wake-up frequency assignment problem. 
The rest of the paper is organized as follows. In Section II we present related work. Section III describes the energy expenditure model we consider. In Section IV we study the problem of $\tau / D$-optimal wake-up frequency assignment in multiple trees and present two algorithms: an optimal one and an approximation. In Section $\mathrm{V}$ we study the problem of constructing an efficient routing tree. We prove that the problem is NP-hard and propose an approximation algorithm. In Section VI we present simulation results and Section VII concludes the paper.

\section{RELATED WORK}

The problem of energy efficient routing in sensor and adhoc networks is a well-known and multifaceted problem. This problem has different solutions in synchronous and asynchronous networks. A nice solution for a synchronous network with one gateway is proposed by the DMAC protocol [14]. The main idea here is that the sensors wake up sequentially, like a chain reaction. An interval of each sensor is divided into receiving, sending, and sleep periods. In receiving state, the node is expected to receive a packet while in sending state it sends the packet to the next hop towards the gateway. The receiving and sending periods have same length, which is enough for one packet transmission and reception.

The model considered by DMAC has two limitations. First, all the sensors must be synchronized to the same time. Such global synchronization is inefficient and very difficult, or expensive, to achieve in big sensor networks [2]-[4]. Another problem is the fact that the wake-up periods of all the sensors are of equal and predefined length. Thus, such a protocol cannot support many sensor network applications, which generate non-uniform traffic. In this paper we consider an asynchronous sensor network, where the receive period of every sensor $u$ is independent of the receive period of $u$ 's neighbors, and its length can be determined locally by $u$ according to the actual load imposed on it. In [7], an algorithm for maximizing the lifetime of a such a sensor network while guaranteeing an upper bound on the end-to-end delay is presented. The paper considers a wireless sensor network with a routing spanning tree already constructed. The goal is to determine the wakeup frequency of each node such that an upper bound on the routing delay is achieved while the network lifetime is maximized.

Energy efficiency can be achieved in different ways. For example, energy aware routing finds a routing path while taking into account energy cost and energy available at the sensors along the route. In [15], the number of hops along the forwarding path is considered, while keeping in mind that transmission between close nodes is more energy efficient. The authors use a random network model to show the dependency between energy, latency and throughput, and to find an optimal transmission power assignment.

The tradeoff between energy and latency in general wireless networks was also studied in various contexts. For example, [16] and [17] investigate this tradeoff when a technique called "modulation scaling" is used. The authors base their work on the observation that in many coding schemes the transmission of a packet requires a smaller amount of energy if it lasts longer. They solve the problem of finding an optimal transmission schedule for a node, given that it has to forward a random number of packets whose arrival times follow a Poisson distribution. The optimization criterion is to minimize the overall energy expenditure and to bound the maximum delay. Two algorithms are proposed: an offline algorithm, which finds an optimal solution, and an online algorithm, which approximates the optimal solution. This approach is taken further in [18], where packets may have different deadlines and sizes.

In the present paper a sensor network with multiple gateways is also studied. Two operation models can be considered for multiple gateway networks. In the first model, each sensor is associated with one gateway, whereas in the second model, assumed in this paper, every node can send data to any gateway. The second model is studied in the family of SPIN adaptive protocols [12]. In these protocols a sensor broadcasts a special "advertisement" with a description of the data it has, and an interested gateway returns a request for this information. Another algorithm that addresses sensors communicating with multiple gateways is SAR [13]. This algorithm creates multiple trees, where each root is one hop away from the gateway and most of the nodes belong to multiple trees.

\section{ENERGY EXPENDITURE MODEL}

In order to extend the network's lifetime, nodes remain in a sleep mode most of the time, while adhering to the following two simple rules:

(R1) Each node $v$ wakes up periodically in order to receive packets from its neighbors. These neighbors know when $v$ switches from sleep to active mode. A node remains active as long as it receives packets from its neighbors.

(R2) Every node $v$ that has packets for its neighbor $u$ wakes up during $u$ 's duty cycle and sends these packets.

A node that happens to be a leaf in all the spanning trees does not enter the active mode according to a predefined schedule (R1), but only when it needs to forward a packet (R2). This is because packets are always forwarded towards a root.

Following the above rules, if a node $v$ wakes up $f_{v}$ times per second, its energy expenditure is equal to $f_{v} \cdot c_{v}+b_{v}$ (watts), where

- $c_{v}$ is the average amount of energy expended during every wake-up period (R1), including charging registers, listening, receiving, and transmitting packets.

- $b_{v}$ is a constant amount of energy, expended during a time unit on monitoring the environment, performing internal calculations, managing the clock, and forwarding packets to the next node on route to the gateway (R2). It is a constant because it does not depend on $f_{v}$ and is not subject to optimization. Hence, it is often ignored in our model. 


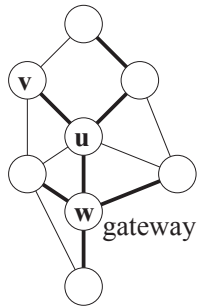

(a) a sensor network

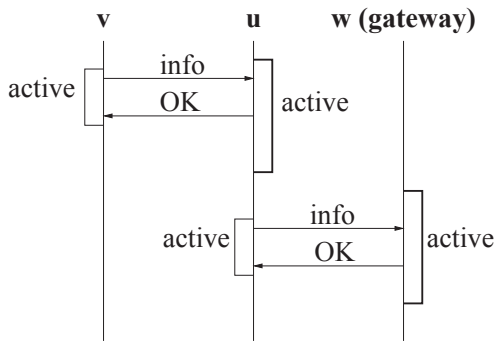

(b) message exchange
Fig. 1. A sensor network (the routing paths to the gateway are marked by thick lines) and a possible packet exchange for delivering data from $v$ to $w$

Our energy expenditure model is justified in light of the significant difference between the energy expended by the sensor radio for activities related to (R1) and the energy expended in sleep mode. For instance, the TI's CC2420 device [19]-[21], which is 802.15.4-compatible, expends $52.2 \mathrm{~mW}$ for transmitting, $56.4 \mathrm{~mW}$ for receiving or listening, and only $3 \mu \mathrm{W}$ for sleeping. A typical duty cycle of such a sensor is $0.1 \%$. The difference between the energy required for receiving/listening $(56.4 \mathrm{~mW})$ and transmitting $(52.2 \mathrm{~mW})$ is negligible compared to the difference between receiving/listening/transmitting (52$56 \mathrm{~mW})$ and sleeping $(3 \mu W)$. Hence, while the actual energy expended by the sensor is $0.001 \cdot 56+0.999 \cdot 0.003+b_{v} \mathrm{~mW}$, it can be very well approximated by $0.001 \cdot 56+0.003+b_{v}$ $\mathrm{mW}$. Thus, by incorporating the fixed part of the energy spent in the sleep mode $(0.003 \mathrm{~mW})$ into $b_{v}$, we get that the average expended energy is indeed $f_{v} \cdot c_{v}+b_{v} \mathrm{~mW}$, where $b_{v}$ does not depend on the wake-up frequency $f_{v}$.

There is an obvious correlation between the energy expended by the nodes and the delay experienced by routing of messages: more frequent wake-ups (and greater expended energy (R1)) result in a shorter delay for a message waiting to be forwarded from one node to another. Therefore, we not only seek an upper bound on the end-to-end delay, but also aim at minimizing the energy expended by the nodes and enforcing an upper bound on the energy expended by each one.

Figure 1 depicts the considered communication model for the case where sensor $v$ sends a packet to the gateway $w$ via sensor $u$. Node $v$ wakes up during the duty cycle of node $u$, and sends a packet to $u$. The energy it expends for transmitting the packet to $u$ is attributed to its $b_{v}$ factor, whereas the energy expended by $u$ is attributed to its $f_{u} \cdot c_{u}$ factor. Node $u$ wakes up during the duty cycle of $w$ and forwards the packet to $w$. The energy it expends for transmitting the packet is attributed to its $b_{v}$ factor. The end-to-end delay encountered by this packet is the time node $v$ waits until $u$ wakes up, plus the time node $u$ waits until node $w$ wakes up. This sum is upper bounded by $1 / f_{u}+1 / f_{w}$.

Precise synchronization between neighboring nodes is unnecessary in the considered model. If the nodes use a CSMA/CA-like MAC protocol in order to send data packets, a packet that is not ACKed due to imperfect synchronization will be retransmitted after a short time-out period, before the receiving node returns to sleep mode.

Following rule (R1), not all the nodes need a wake-up frequency assignment. If a node is a leaf in all spanning trees, it wakes up only when it needs to send a packet to one of its parents. Therefore, $f_{v}=0$ holds for such a node, and all the energy it expends is attributed to its $b_{u}$ component. A node $v$ that is not a leaf for one or more trees must have $f_{v}>0$.

Both the energy expenditure of a sensor network and the end-to-end delay of a packet depend on the wake-up frequency of the nodes [7]. However, in order to increase the network's lifetime, it is not enough to minimize the energy expenditure. Maximal node energy expenditure should also be taken into account. It may be sufficient to bound it, even by increasing the total energy expended. Hence, we seek an optimal wakeup frequency assignment in light of the two main constraints. To this end, we use the following definition from [7]:

Definition 1: A $\tau / D$-optimal wake-up frequency assignment to nodes is an assignment that guarantees an upper bound $\tau$ on the energy expended by every node and an upper bound $D$ on the maximum delay, while minimizing the total energy expended by the nodes in active mode.

\section{THE MT-OFA PROBLEM}

The MT-OFA problem is to find a $\tau / D$-optimal wake-up frequency assignment for a given network with multiple trees. This problem is formally defined as follows.

Problem 1: (MT-OFA problem):

Given a graph $G=(V, E)$, a set of trees $\left\{T_{1}, T_{2}, \ldots, T_{n}\right\}$, upper bounds $\left\{D_{1}, D_{2}, \ldots, D_{n}\right\}$ on the end-to-end delay in each tree, an upper bound $\tau$ on the energy expended by every node due to (R1), and a function $C: V \rightarrow Z^{+}$that defines the energy expended by every node per wake-up, assign to each node $v$ a wake-up frequency $f_{v}$ such that

1) $\forall v \in V, f_{v} \cdot c_{v} \leq \tau$; i.e, the energy expended by every node due to (R1), is upper bounded by $\tau$.

2) For every tree $T_{i}$ and for every path $P \in T_{i}, \sum_{v \in P} \frac{1}{f_{v}} \leq$ $D_{i}$; i.e, the end-to-end delay on each directed path in every tree $T_{i}$ is bounded by $D_{i}$.

3) $\sum_{v \in V} f_{v} \cdot c_{v}$ is minimized.

The special case of a single gateway and a single routing tree is studied in [7], where an optimal polynomial time algorithm for this problem is presented. The algorithm performs several iterations over the tree. During each iteration it computes an optimal wake-up frequency assignment for the nodes of some sub-trees while restricting the maximum wakeup frequency. In the rest of this paper, this algorithm is referred to as the optimal ST-OFA (Single Tree Optimal Frequency Assignment) algorithm.

In its general form, MT-OFA is a convex optimization problem. To see why, note that the delay through a single node is a convex function of the wake-up frequency. The delay on a path $\mathrm{P}$ is a sum of convex functions and, therefore, is a convex function as well. Finally, the target function is linear in $f_{v}$. 
We now reformulate MT-OFA into a conic quadratic optimization problem, which has only linear and convex cone constraints. The reciprocals $\frac{1}{f_{v}}$ are replaced by new variables $d_{v}$, which are not part of the target function, and for every $v$ we add a constraint $d_{v}=\frac{1}{f_{v}}$. This problem can be solved using the interior point method [22], by traversing the interior values of the problem (unlike the simplex method in which the edges are traversed), until it reaches the maximum. It is shown in [23] that the optimal solution is found after a polynomial number of iterations. The resulting optimal algorithm for MTOFA is:

Algorithm 1: (Optimal Algorithm for MT-OFA):

Use the interior point method to minimize $\sum_{v \in V} f_{v} \cdot c_{v}$, subject to:

1) $\forall v \in V, f_{v} \cdot c_{v} \leq \tau$; i.e, the energy expended by every node due to (R1) is upper bounded by $\tau$.

2) For every tree $T_{i}$ and for every path $P \in T_{i}, \sum_{v \in P} d_{v} \leq$ $D_{i}$; i.e, the end-to-end delay on each directed path for each tree $T_{i}$ is bounded by $D_{i}$.

3) $\forall v \in V, d_{v}=1 / f_{v}$.

4) $\forall v \in V, f_{v}, d_{v} \geq 0$.

Algorithm 1 is impractical in many cases because it demands high computational power and because it requires centralized processing by a node that has a complete network view. Therefore, we now propose an efficient approximation for MT-OFA that can be distributedly implemented. This approximation uses the ST-OFA algorithm as a sub-routine.

Algorithm 2: (An approximation algorithm for MT-OFA):

1) Execute the ST-OFA algorithm for each tree $T_{i}$, and find the wake-up frequency $F_{i}(v)$ assigned to every node $v$ for each tree $T_{i}$

2) Assign to each node $v$ a wake-up frequency $F(v)=$ $\max F_{i}(v)$.

If this algorithm is performed by a centralized node, its time complexity for the first step is $O(n|V|)$ and for the second step is $O(|V|)$, where $n$ is the number of trees and $|V|$ is the number of nodes in the graph. For distributed implementation, Algorithm 2 requires to run ST-OFA independently for each tree, which takes $O\left(V^{2}\right)$ time. Thus, the total time/message complexity for distributed implementation is $O\left(n \cdot V^{2}\right)$.

Theorem 1: A solution found by Algorithm 2 is feasible because it fulfills the first two constraints in Problem 1. In addition, the total energy expended by the network nodes is at most $n$ times greater than that of the optimal solution, where $n$ is the number of trees.

Proof: Let $F^{*}$ be the wake-up frequency vector computed by Algorithm 2, and let $F_{1}, F_{2}, \ldots F_{n}$ be the wake-up frequency vectors computed by the optimal ST-OFA algorithm for each individual tree. For each vector $F$, let $F(v)$ denote the wake-up frequency assigned to node $v$ and $|F|=\sum_{v \in V} c_{v}$ the energy expenditure of the network under the wake-up frequency assignment $F$.

For every node $v$ and for every $i=1, \ldots, n, F^{*}(v) \cdot c_{v} \leq \tau$. Hence, the first constraint clearly holds. Since the delay on any path $P$ under $F^{*}$ is not greater than the delay under any other assignment $F_{i}$, the second constraint holds as well.
To prove the second part, note that $\left|F^{*}\right|=$ $\begin{array}{lccc}\sum_{v \in V} c_{v} F^{*}(v) & < & \sum_{v \in V} c_{v} \sum_{1 \leq i \leq n} F_{i}(v) & = \\ \sum_{1 \leq i \leq n} \sum_{v \in V} c_{v} F_{i}(v) & = & \sum_{1 \leq i \leq n}\left|F_{i}\right| & \leq\end{array}$ $n \max _{1 \leq i \leq n}\left|F_{i}\right| \leq n F_{O P T}$, where $F_{O P T}$ is the optimal wake-up frequency assignment.

\section{Constructing an Energy-Efficient Tree}

ST-OFA and MT-OFA are defined for an already constructed spanning tree (or set of spanning trees). In this section we address the problem of building energy efficient trees in advance.

We first formally define the problem and then prove that it is NP-hard even for a single tree.

Problem 2: (The Minimum Energy Tree Problem (METP)): Given a graph $G=\{V, E\}$, a node $r$, and a function $C: V \rightarrow$ $Z^{+}$that defines the energy expended by every node during every wake-up, find a spanning tree rooted at $r$ for which there exists a $\tau / D$-optimal frequency assignment whose total energy expenditure is minimal. This tree is referred to as the best $\tau / D$-optimal tree.

Claim 1: Consider the best $\tau / D$-optimal tree in a graph $G=(V, E)$. Let $T$ be this tree without the leaves. Then, $T$ contains no pair of nodes $u$ and $v$ such that $(u, v) \in E$, $(u, v) \notin T$ and either $u$ is a descendant of $v$ in $T$ or vice versa.

Proof: Assume by contradiction that such a pair of nodes exists. Without loss of generality, let $u$ be a descendant of $v$. Let $T^{\prime}$ be the same as $T$, except that the sub-tree of $u$ is directly connected to $v$. That is, we remove from $T$ the edge between $u$ and its parent and add an edge $(u, v)$. We now show that a $\tau / D$-optimal frequency assignment for $T^{\prime}$ is better than a $\tau / D$-optimal frequency assignment for $T$. Consider a $\tau / D$-optimal frequency assignment for $T$. If we use the same assignment for $T^{\prime}$, the delay from each node in the sub-tree of $u$ to the root in $T^{\prime}$ is smaller than in $T$ because the path from $u$ to the root in $T^{\prime}$ is a sub-path of the corresponding route in $T$. Hence, we can decrease the wake-up frequency and therefore the energy expended by some of the nodes in the sub-tree of $u$ without increasing the maximum latency beyond $D$. Thus, $T^{\prime}$ is a better $\tau / D$-optimal spanning tree than $T$, in contradiction to our assumption.

Claim 2: The overall energy expenditure of a tree whose structure without the leaves is a star under a $\tau / D$-optimal wake-up frequency assignment is $\left(\sqrt{c}+\sqrt{\sum_{i=1}^{n} c_{i}}\right)^{2}$ if $\tau$ is infinite and $D=1$. Here, $c$ and $c_{1}, \ldots, c_{n}$ are the amounts of energy expended per wake-up by $v$ and $v_{1}, \ldots, v_{n}$ respectively. Proof: Note that in the following analysis we ignore the constant components $b_{v}$, since their values are not affected by the tree structure. Let $v$ be the root and $v_{1}, \ldots, v_{n}$ be the leaves of the inner tree. The energy expended per time unit for $v$ is $e$ and for $v_{1}, \ldots, v_{n}$ it is $e_{1}, \ldots, e_{n}$. Following this notation, the wake-up frequency of $v$ is $c / e$, and the wake-up frequency of every other node $v_{i}$ is $c_{i} / e_{i}$. In order to solve the ST-OFA problem, the following set of $n+1$ equations has 
to be solved [7]:

$$
\begin{gathered}
E=e+e_{1}+e_{2}+\ldots+e_{n} \\
\frac{c_{1}}{e_{1}}=\frac{c_{2}}{e_{2}}=\ldots=\frac{c_{n}}{e_{n}} \\
\frac{\delta\left(\frac{c}{e}+\frac{c_{1}}{e_{1}}\right)}{\delta e}=0 .
\end{gathered}
$$

In the first equation $E$ is the total energy expended by all the nodes in the tree. The next $n-1$ equations dictate that the delay on the paths from the leaves to the root are equal and the last equation dictates that this delay is minimal, because, as shown in [7], minimizing the delay for a given $E$ is equivalent to minimizing the energy expenditure for a given delay.

From this set of equations we get that for every $i, 1 \leq i \leq n$, $e_{i}=\left(e_{1} / c_{1}\right) \cdot c_{i}$.

Hence,

$$
E=e+\sum_{i=1}^{n} \frac{e_{1}}{c_{1}} c_{i}=e+\frac{e_{1}}{c_{1}} \sum_{i=1}^{n} c_{i} .
$$

Therefore,

$$
e_{1}=\frac{E-e}{\frac{\sum_{i=1}^{n} c_{i}}{c_{1}}}
$$

and

$$
\frac{\delta \frac{c}{e}+\frac{c_{1}}{\frac{E-e}{\sum_{i=1}^{n} c_{i}}}}{\delta e}=-\frac{c}{c_{1}}+\frac{\sum_{i=1}^{n} c_{i}}{(E-e)^{2}}=0 .
$$

We then get that

$$
\begin{aligned}
-c(E-e)^{2}+e^{2} \sum_{i=1}^{n} c_{i} & = \\
\left(\sum_{i=1}^{n} c_{i}-c\right) e^{2}+2 E c e-c E^{2} & =0
\end{aligned}
$$

and

$$
\begin{aligned}
e & =\frac{-2 E c \pm \sqrt{4 E^{2} c^{2}+4 E^{2} c\left(\sum_{i=1}^{n} c_{i}-c\right)}}{2 \sum_{i=1}^{n} c_{i}-c} \\
& =E \frac{-c+\sqrt{c \sum_{i=1}^{n} c_{i}}}{\sum_{i=1}^{n} c_{i}-c} .
\end{aligned}
$$

Therefore, the delay on the tree is

$$
\frac{c}{e}+\frac{c_{1}}{e_{1}}=\frac{\left(\sqrt{c}+\sqrt{\sum_{i=1}^{n} c_{i}}\right)^{2}}{E} .
$$

Taking $D$ to be equal to 1 , we have

$$
E=\left(\sqrt{c}+\sqrt{\sum_{i=1}^{n} c_{i}}\right)^{2} .
$$

Note that for the case where every $c_{v}=1$, the overall energy expenditure of the tree is $E=1+2 \sqrt{n-1}+n-1=n+$ $2 \sqrt{n-1}$.

Claim 3: METP is NP-hard even in the special case where the energy expended by each node is equal to $f_{v}$, namely, $\forall v \in V, c_{v}=1$.
Proof: We prove this using a reduction to the well-known NP-hard minimum dominating set (MDS) problem [24]. The exact details are omitted due to lack of space.

We now identify the best and the worst $\tau / D$-optimal trees for ST-OFA. This will help us to develop an approximation algorithm for METP.

Claim 4: For a given set of nodes that should build the inner tree $T$, the tree with the best $\tau / D$-optimal wake-up frequency assignment has a star topology.

Proof: Follows from Claim 1.

Claim 5: For a given set of nodes that should build the inner tree $T$, the tree with the worst $\tau / D$-optimal wake-up frequency assignment is a cascade.

Proof: Let $F$ be the wake-up frequency assignment to the nodes of the cascade tree and $P$ the path from the leaf to the root in $T$. Consider a non-cascade tree $T^{\prime}$ built over the same graph using the same nodes. We assign a wake-up frequency to the nodes of $T^{\prime}$ according to $F$. Since any path $P^{\prime}$ in $T^{\prime}$ is a sub-path of $P$, the overall delay on $P^{\prime}$ is smaller than the maximum tolerated delay $D$. Hence, the wake-up frequency assigned according to $F$ to $T^{\prime}$ can be improved, thereby making $T^{\prime}$ a better $\tau / D$-optimal tree. We conclude that any non-cascade tree, consisting of the same set of nodes, is a better $\tau / D$-optimal tree than $T$, which implies that $T$ is the worst $\tau / D$-optimal tree.

Algorithm 3: (an approximation for METP):

Let the input for the METP problem be a graph $G$ and a function $C: V \rightarrow Z^{+}$that defines the energy expended by every node during each wake-up. Let $G$ and $C$ be also the input of the minimum-weighted connected dominating set (MWCDS) problem, where $C$ in this case is used as a weight function. Let $V^{\prime}$ be a solution for the MWCDS problem. We create a directed spanning tree in $G$ whose inner nodes are $V^{\prime}$. If several trees can be created, we pick one of them arbitrarily.

Claim 6: The selected tree $T$ is an $n^{\prime}$-approximation for METP, where $n^{\prime}$ is the size of a minimum-weighted connected dominating set $V^{\prime}$.

Proof: Let us find a lower bound on the energy expenditure of a spanning tree in $G$. By Claim 2, for any tree $T^{\prime}$ the smallest energy expenditure is achieved if the inner tree of $T^{\prime}$ is a star. In this case, the energy expenditure is at least $\sum_{v \in T_{\text {inner }}^{\prime}} c_{v}$. We seek to minimize this sum by careful selection of the inner tree nodes. Since the inner tree is a connected dominating set, the minimum of $\sum_{v \in T_{i n n e}^{\prime}} c_{v}$ is obtained for a minimumweighted dominating set $V^{\prime}$. That is, $\sum_{v \in V^{\prime}} c_{v}$ is a lower bound on the energy expended by any tree. By reducing the value of $\tau$, the tree total energy expenditure can only increase. Hence, $\sum_{v \in V^{\prime}} c_{v}$ is a lower bound on the energy expenditure for any $\tau$.

Although the actual energy consumption of $T$ depends on the graph topology, we can still find an upper bound on the energy expended by its nodes. By Claim 5, the worst case occurs when the inner tree is a cascade tree. We now present a feasible wake-up frequency assignment, assuming that $\tau$ is infinite. We choose the wake-up frequencies so that the energy expended by all the nodes is equal, i.e., $c_{v} f_{v}=e$ 
for every $v \in G$. In order to find the value of $e$, note that the delay imposed by each node is equal to $c_{v} / e$. Hence, the delay on the path from the inner tree leaf to the root is $n^{\prime} \sum_{v \in T_{\text {inner }}} \frac{c_{v}}{e}=D$, where $n^{\prime}$ is the path length (i.e., the number of nodes in the inner tree of $T$ ). Taking $D=1$, we get that $e=\sum_{v \in T_{\text {inner }}} c_{v}$ and the overall energy expended by this tree is $n^{\prime} \sum_{v \in T_{\text {inner }}} c_{v}$.

Next, we show that the above expression is an upper bound on the total energy expenditure for any value of $\tau$. Obviously, for certain values of $\tau$ this solution is not feasible because $e>\tau$. However, in this case no feasible solution for the cascade-type inner tree exists, and any feasible solution on any other tree has a total energy expenditure smaller than $n^{\prime} \sum_{v \in T_{\text {inner }}} c_{v}$.

To summarize, we obtained an upper bound on the energy expenditure of a spanning tree $T$ constructed from $V^{\prime}$. This upper bound is $n^{\prime}$ times greater than the lower bound obtained for any spanning tree. Note that in the proof we implicitly assumed that $r \in V^{\prime}$. However, since $r$ must be part of the inner tree both in the optimal and the approximated solutions, we can take its weight to be 0 . Consequently, $r$ can be added to any solution without affecting the energy expenditure.

The minimum-weighted connected dominating set (MWCDS) problem is a generalization of the minimum connected dominating set (MCDS) problem, and is therefore NP-hard [24]. In the general case, MWCDS has an approximation algorithm with factor $O(\log n)$ [25], where $n$ is the number of nodes. However, for the unit disk graphs, which are also known as geometric graphs, there exist algorithms with a constant approximation factor [26].

\section{Simulation Study}

\section{A. Simulation Results for MT-OFA}

To study the actual performance of the algorithm presented in Section IV for MT-OFA, we use the simulation model presented in earlier works [27], [28] as follows. We randomly place sensors over a $100 \times 100$ grid. We vary the transmission range between $20 \%$ and $40 \%$ of the grid size. For each range $r$, any two nodes whose Euclidean distance is not greater than $r$ are considered to be connected. The gateway nodes are randomly selected as well. If the created graph is not connected, this simulation instance is ignored.

We start with comparing the performance of several wakeup assignment algorithms for a given tree. The trees are built using a BFS algorithm. However, we obtained similar results when we used other algorithms for building the tree. The number of trees varies between 2 and 10. We find the optimal solution by Algorithm 1 and compare it to the approximation proposed by Algorithm 2. Recall that the energy expenditure of a node $v$ is $f_{v} c_{v}+b_{v}$, where $c_{v}$ is the energy expended during every wake-up period. However, the nodes are assumed to have a similar per wake-up energy expenditure, namely, $c_{v}=1$ holds for every $v$. The value of $\tau$ is taken to be infinite and the upper bound $D$ on the end-to-end delay is 100 time units for every tree.

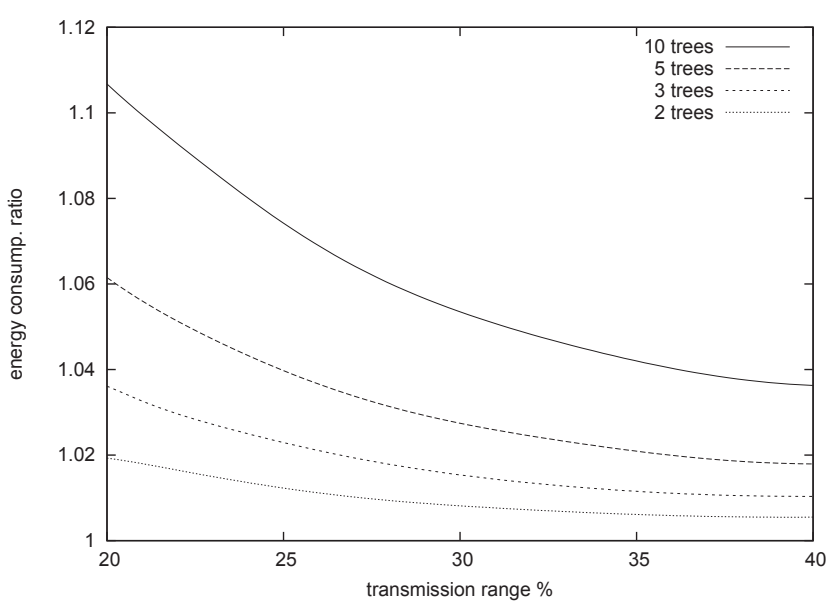

Fig. 2. Approximation algorithm results for multiple trees

In order to take advantage of available tools for finding an optimal solution using Algorithm 1, another relaxation is made to the MT-OFA problem: the constraint $d_{v} f_{v}=1$ is replaced by $d_{v} f_{v} \geq 1$. Since we solve a minimization problem where all the coefficients in the target function are positive, the function receives its minimum only when all of the $f_{v}$ values receive their minimum. This happens when $d_{v} \cdot f_{v}$ is exactly 1 . Hence, these two constraints are equivalent. In its new form, the problem represents a conic constraint optimization problem. The target function is linear and the first set of constraints is linear as well. The second set of constraints represents a rotated quadratic cone. To calculate the optimal solution, we use the MOSEK tool.

For the implementation of Algorithm 2, the wake-up frequency for each node is calculated for every tree using the optimal ST-OFA algorithm. Then, each node is assigned the maximum frequency value. Finally, we compare the ratio of the overall energy expenditure of Algorithm 2 and the overall energy expenditure of the optimal solution obtained by Algorithm 1.

Figure 2 presents the simulation results for 2-10 spanning trees in networks with 2000 nodes. The graphs show the performance ratio as a function of the transmission range. For each transmission range and number of trees, the simulation is conducted 50 independent times on randomly created instances. Then, the average value is calculated and plotted. We can see that for every number of trees, the actual performance of Algorithm 2 is much better than the worst-case theoretical performance. For 2000 nodes (Figure 2), this ratio ranges between 1.02 for 2 trees and 1.12 for 10 trees, while the theoretical ratio ranges between 2 and 10 . Thus, we believe that the approximation algorithm introduces an excellent combination of good performance and low complexity.

We can see that the relative performance of Algorithm 2 improves when the transmission range increases, because when it does, more and more nodes of each tree are leaves, and the percent of inner nodes in the tree decreases. In this case, 


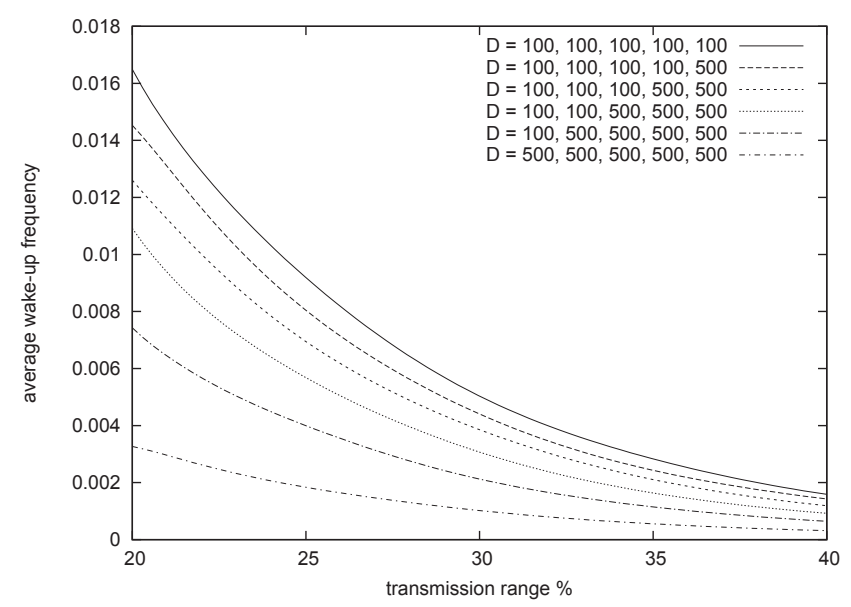

Fig. 3. Average wake-up frequency for different $D_{i}$ values

most of the nodes are either not part of any inner tree or belong to no more than one of the inner trees. In the former case the nodes have a 0 wake-up frequency under both algorithms. In the latter case the nodes are assigned the same (non-zero) wake-up frequency under both algorithms.

In Figure 3 we study the effect of assigning a different upper bound $D$ on the end-to-end delay to five different trees. For some of the trees $D=100$ while for the others $D=500$. The upper curve shows the average node wake-up frequency when all the trees have $D=100$. The second curve from the top shows the case when 4 trees have $D=100$ and one has $D=500$, the third when 3 trees have $D=100$, the fourth when 4 trees have $D=100$, and the fifth when 5 trees have $D=100$. As expected, it can be seen from the upper curve that the average wake-up frequency of the first tree is approximately 5 times greater than that of the last one. We also observe that the gaps between the upper curves are smaller than those of the lower curves. This can be explained by the fact that the inner trees are not completely disjoint, but have a small number of common nodes. These nodes are assigned the maximum wake-up frequency among all 5 assignments.

\section{B. METP Approximation Simulations}

To study the efficiency of the tree construction algorithm, we use similar settings to those described in Section VI-A. We build a sensor network by randomly placing 1,000 nodes in a $100 \times 100$ grid. The transmission range varies between $15 \%$ and $50 \%$ of the whole grid. For each range $r$, any two nodes whose Euclidean distance is not greater than $r$ are considered to be connected. If the created graph is not connected, this instance is ignored. If it is connected, we select a random node to function as the gateway. The nodes are assumed to have similar per wake-up energy expenditure: namely, for every node $v$, $c_{v}=1$. The value of $\tau$ is taken to be infinite and the upper bound $D$ on the end-to-end delay is 100 time units.

We compare a BFS tree to Algorithm 3. Because we take $c_{v}=1$ for every $v$, we can approximate METP using a minimum connected dominating set (MCDS) rather than a minimum-weighted connected dominating set. An MCDS is constructed using the algorithm presented in [29].

For each simulation instance we perform the following steps:

1) Build two spanning trees: one by Algorithm 3 and another by a BFS algorithm.

2) Invoke the ST-OFA algorithm on each tree in order to assign optimal wake-up frequencies to the nodes.

Figure 4(a) shows the average wake-up frequency of the nodes as a function of the transmission range for the two spanning trees. It turns out that the average wake-up frequency assigned to a tree built by Algorithm 3 is half of the value assigned to a BFS tree. This indicates that even if an optimal algorithm is used for wake-up frequency assignment, it is crucial to optimize the tree structure. Figure 4(b) shows the number of inner nodes in both trees. It is evident that the relation between the inner tree sizes is similar to the relation between the average wake-up frequency assignment. This indicates that an efficient tree is indeed one whose set of inner nodes is minimal.

Finally, carry out combined simulations. In one instance, multiple trees are created on a random graph using Algorithm 3 , and wake-up frequencies are assigned using Algorithm 2. In another instance, the trees are constructed using a BFS algorithm, and the wake-up frequencies are again assigned using Algorithm 2. Figure 5(a) shows the simulation results for the BFS tree and Figure 5(b) for the tree constructed by Algorithm 3. It is evident that for every number of trees, Algorithm 3 significantly reduces the average wake-up frequency of the nodes.

\section{Simulating Efficiency of Multiple Spanning Trees}

The main advantage of using multiple trees is improved reliability. In a single routing tree, neighboring nodes that discover an event usually follow very similar paths to the gateway. Hence, a single failure may disconnect all the reporting nodes from the gateway. However, if several routing trees are used, and each node randomly chooses a gateway, at least some of the messages are likely to reach a one of the chosen gateways even if a failure occurs. In Figure 6(a) we compare this aspect of reliability for a single and for multiple gateways. In this simulation study, one or more trees are constructed on a random graph. We simulate the worst case, which is the failure of a node adjacent to a gateway. These nodes are likely to have more descendants than other nodes. We randomly choose one of the trees, denoted by $T$, whose root is the gateway $g$. We then randomly choose a node $v$ adjacent to $g$, and remove all $v$ 's edges from the graph. Consequently, all the descendants of $v$ in $T$ are disconnected from $g$. If multiple trees are used, each of these disconnected nodes is likely to have a path to another gateway. Figure 6(a) shows the percent of nodes that have no gateway connectivity for 1,2 and 3 trees. We can see that for 2 routing trees the number of disconnected nodes is dramatically reduced. For 3 trees this number is often 1, which indicates that only the failed node loses connectivity. 


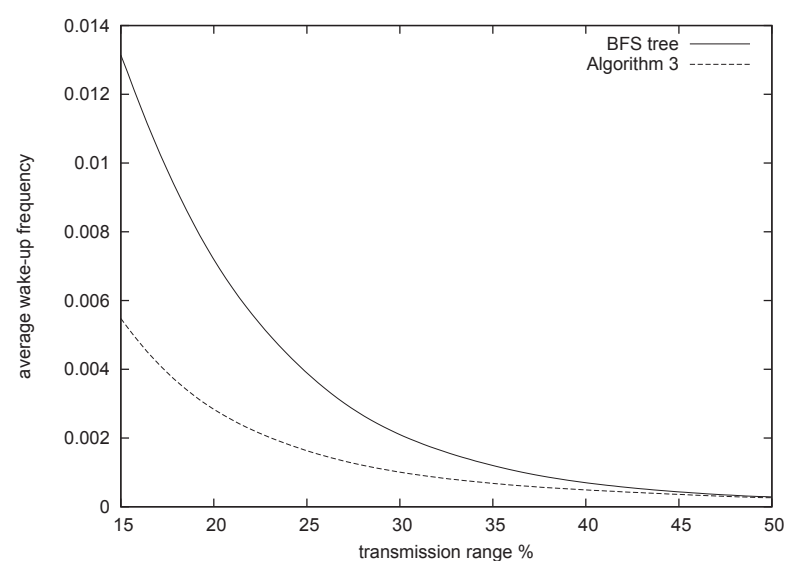

(a) the average wake-up frequency

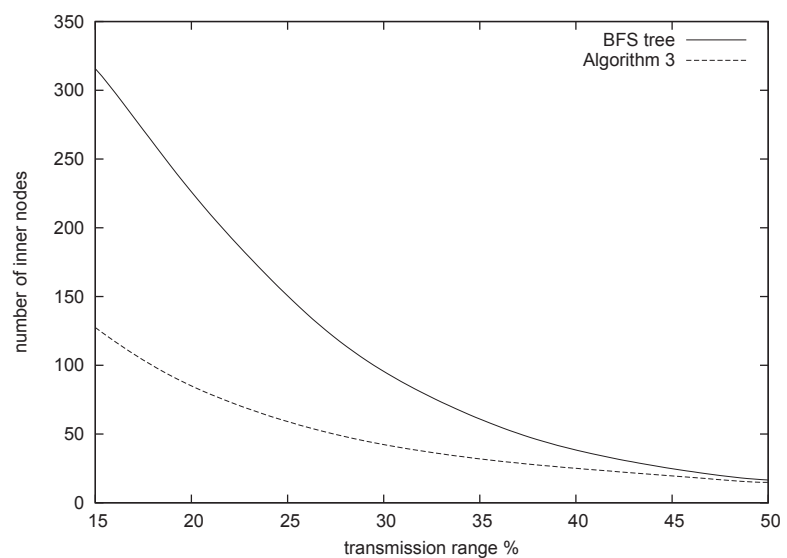

(b) number of inner nodes

Fig. 4. Wake-up assignment to nodes of a tree constructed by Algorithm 3 and a tree constructed by a BFS algorithm

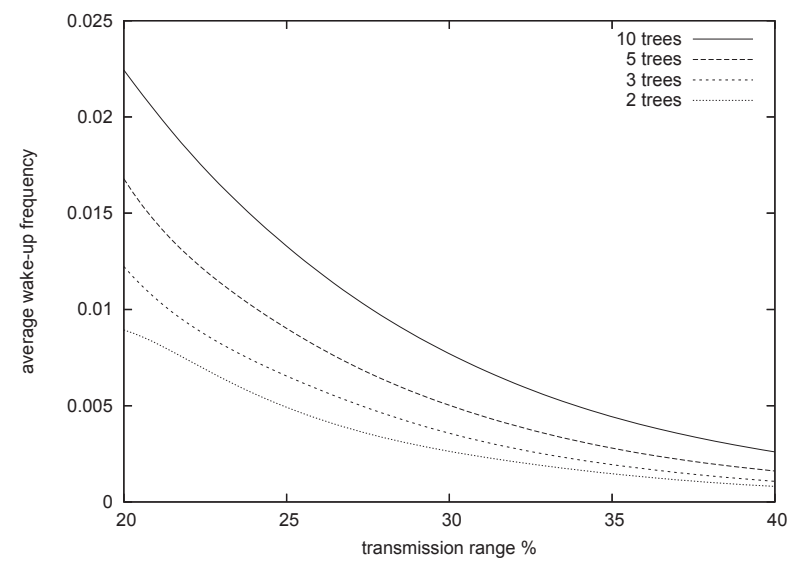

(a) BFS tree

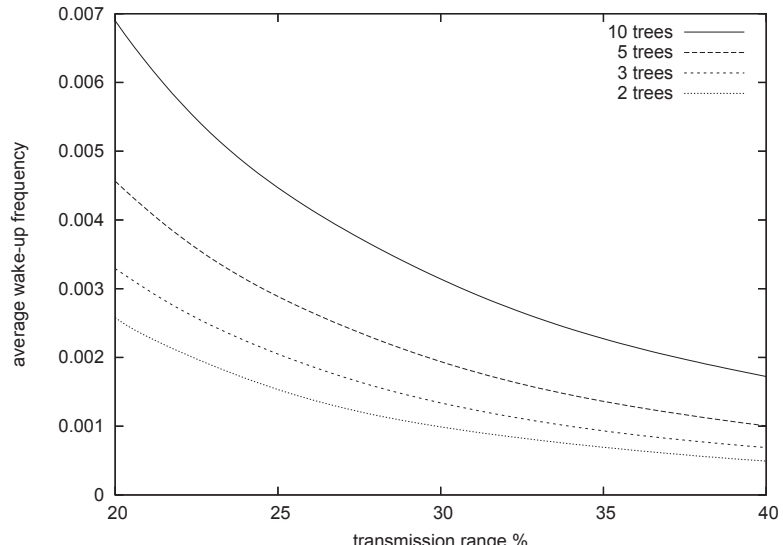

(b) METP tree

Fig. 5. Comparing two $\tau / D$-optimal trees

Creating and maintaining multiple trees is more energy demanding than a single tree. However, the various algorithms proposed in this paper reduce this extra energy cost. This is especially true for distant gateways, because energy expenditure in the vicinity of the gateways is greater. This is clearly shown in Figure 6(b), where we simulate the network lifetime for 15 spanning trees to which wake-up frequencies are assigned using Algorithm 2. Each node is equipped with some initial amount of energy, which allows the node to be active for some fixed time duration. After every time unit, the energy of each node is reduced according to the node's wake-up frequency. A node whose energy runs below $20 \%$ of its initial value can no longer act as an inner node. Therefore, new trees are built, for which every inner node has at least $30 \%$ of its initial energy. If a spanning tree cannot be rebuilt, the network is considered "dead."

Figure 6(b) shows the ratio between the average lifetime of a network with a single gateway and networks with 25 gateways, as a function of the transmission range. The extra reliability provided by multiple trees reduces the network lifetime by $35 \%$ for 2 trees and by $57 \%$ for 5 trees.

\section{CONCLUSIONS}

This paper studied the problem of energy efficient routing in a sensor network with multiple gateways. Due to the complexity of this problem, we divided it into two subproblems: the problem of constructing efficient routing trees (METP) and the problem of wake-up frequency assignment in a network with multiple routing trees (MT-OFA).

We showed that MT-OFA can be formulated as a conic quadratic optimization problem that has only linear and convex cone constraints. Thus, it can be solved using the interior point method. However, this algorithm is impractical in many cases because it demands high computational power. Therefore, we also proposed an efficient approximation that uses the optimal ST-OFA algorithm as a sub-routine. For METP, we proved that it is NP-hard, and proposed an approximation algorithm that builds a minimum-weighted connected dominating set.

Our simulations showed that the actual performance of the MT-OFA approximation is very close to the optimal. 


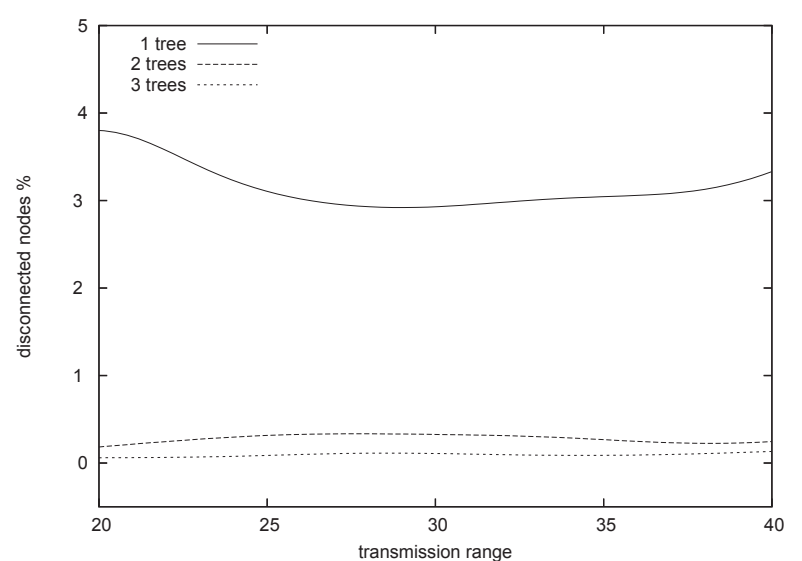

(a) The effect on network reliability

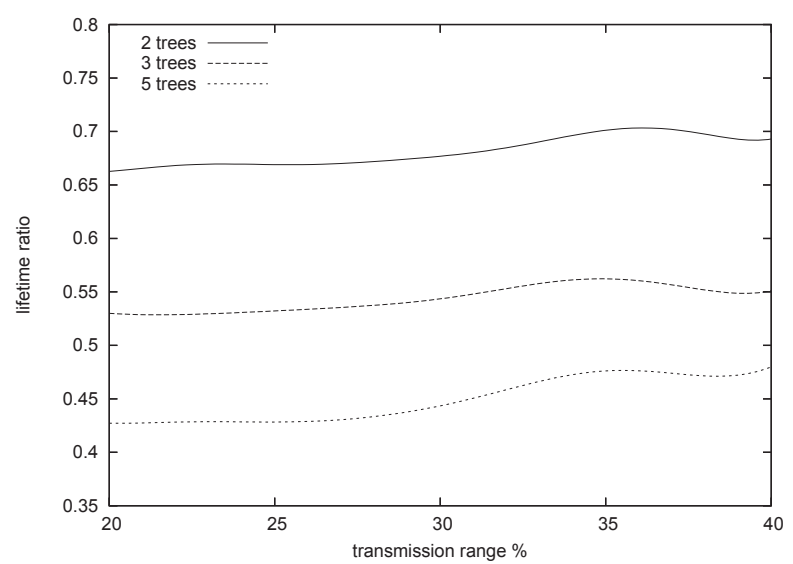

(b) The effect on network lifetime

Fig. 6. The effect of multiple trees

Moreover, when the spanning tree is constructed using the METP approximation algorithm, the wake-up frequencies assigned to the nodes decreases significantly in comparison to the BFS algorithm. Finally, our simulation results show that multiple routing spanning trees significantly improve network reliability.

\section{REFERENCES}

[1] Q. Li and D. Rus, "Global clock synchronization in sensor networks," IEEE Trans. Comput., vol. 55, no. 2, pp. 214-226, 2006.

[2] J. Elson and K. Römer, "Wireless sensor networks: a new regime for time synchronization," SIGCOMM Comput. Commun. Rev, vol. 33, no. 1, pp. $149-154,2003$.

[3] Y. Li, W. Ye, and J. Heidemann, "Energy and latency control in low duty cycle MAC protocols," in Proceedings of the IEEE Wireless Communications and Networking Conference, New Orleans, LA, March 2005, pp. 676-682.

[4] J. Elson, L. Girod, and D. Estrin, "Fine-grained network time synchronization using reference broadcasts," SIGOPS Oper. Syst. Rev., vol. 36, no. SI, pp. 147-163, 2002.

[5] G. Lu, N. Sadagopan, B. Krishnamachari, and A. Goel, "Delay efficient sleep scheduling in wireless sensor networks," in INFOCOM, 2005, pp. 2470-2481.

[6] O. Dousse, P. Mannersalo, and P. Thiran, "Latency of wireless sensor networks with uncoordinated power saving mechanisms," in MobiHoc, 2004, pp. 109-120.

[7] R. Cohen and B. Kapchits, "An optimal algorithm for minimizing energy consumption while limiting maximum delay in a mesh sensor network," in INFOCOM, 2007, pp. 258-266.

[8] V. Annamalai, S. Gupta, and L. Schwiebert, "On tree-based convergecasting in wireless sensor networks," in IEEE Wireless Communications and Networking Conference, vol. 3, 16-20 March 2003, pp. 1942-1947.

[9] Y. Xue and B. Li, "A location-aided power-aware routing protocol in mobile ad hoc networks," in Proc. of IEEE Globecom, 2001.

[10] M. Strasser, A. Meier, K. Langendoen, and P. Blum, "Dwarf: Delayaware robust forwarding for energy-constrained wireless sensor networks," in DCOSS, 2007, pp. 64-81.

[11] A. Keshavarzian, H. Lee, and L. Venkatraman, "Wakeup scheduling in wireless sensor networks," in MobiHoc: Proceedings of the 7th ACM international symposium on Mobile ad hoc networking and computing. New York, NY: ACM Press, 2006, pp. 322-333.

[12] W. R. Heinzelman, J. Kulik, and H. Balakrishnan, "Adaptive protocols for information dissemination in wireless sensor networks," in Mobile Computing and Networking, 1999, pp. 174-185.

[13] K. Sohrabi, J. Gao, V. Ailawadhi, and G. J. Pottie, "Protocols for selforganization of a wireless sensor network," Personal Communications, IEEE [see also IEEE Wireless Communications], vol. 7, no. 5, pp. 1627, 2000.
[14] G. Lu, B. Krishnamachari, and C. Raghavendra, "An adaptive energyefficient and low-latency MAC for tree-based data gathering in sensor networks," WIRELESS COMMUNICATIONS AND MOBILE COMPUT$I N G$, vol. 7, pp. 863 - 875, May 2007.

[15] A. E. Gamal and J. Mammen, "Optimal hopping in ad hoc wireless networks," in INFOCOM, 2006, pp. 1-10.

[16] B. Prabhakar, E. Uysal-Biyikoglu, and A. E. Gamal, "Energy-efficient transmission over a wireless link via lazy packet scheduling," in INFOCOM, 2001, pp. 386-394.

[17] C. Nair, A. E. Gamal, B. Prabhakar, E. Uysal-Biyikoglu, and S. Zahedi, "Energy-efficient scheduling of packet transmissions over wireless networks," in INFOCOM, 2002, pp. 1773-1782.

[18] L. Miao and C. G. Cassandras, "Optimal transmission scheduling for energy-efficient wireless networks," in INFOCOM, 2006, pp. 1-11.

[19] Chipcon Inc., CC2420 data sheet.

[20] H. Cao, K. Parker, and A. Arora, "O-MAC: A receiver centric power management protocol," in ICNP: Proceedings of the Proceedings of the IEEE International Conference on Network Protocols. Washington, DC: IEEE Computer Society, 2006, pp. 311-320.

[21] W. Ye, F. Silva, and J. Heidemann, "Ultra-low duty cycle mac with scheduled channel polling," in SenSys: Proceedings of the 4th International Conference on Embedded Networked Sensor Systems. New York, NY: ACM Press, 2006, pp. 321-334.

[22] D. P. Bertsekas, Nonlinear Programming. Belmont, MA: Athena Scientific, 1995, 2nd edition 1999.

[23] Y. Nesterov and A. Nemirovskii, Interior Point Polynomial Algorithms in Convex Programming. Belmont, MA: Society for Industrial and Applied Math, 1994.

[24] M. Garey and D. Johnson, Computers and Intractability: A Guide to the Theory of NP-Completeness. San Francisco: W.H. Freeman, 1979.

[25] S. Guha and S. Khuller, "Improved methods for approximating node weighted Steiner trees and connected dominating sets," Information and Computation, vol. 150, pp. 57-74, 1999.

[26] C. Ambühl, T. Erlebach, M. Mihalák, and M. Nunkesser, "Constantfactor approximation for minimum-weight (connected) dominating sets in unit disk graphs," in APPROX-RANDOM, 2006, pp. 3-14.

[27] Y. Xu, J. S. Heidemann, and D. Estrin, "Geography-informed energy conservation for ad hoc routing," in Mobile Computing and Networking, 2001, pp. 70-84.

[28] C. Schurgers, V. Tsiatsis, S. Ganeriwal, and M. Srivastava, "Topology management for sensor networks: exploiting latency and density," in MobiHoc: Proceedings of the 3rd ACM International Symposium on Mobile Ad Hoc Networking \& Computing. New York, NY: ACM Press, 2002, pp. 135-145.

[29] B. Gao, Y. Yang, and H. Ma, "An efficient approximation scheme for minimum connected dominating set in wireless ad hoc networks," VTC: Vehicular Technology Conference, vol. 4, pp. 2744-2748, Sept. 2004. 